OPEN ACCESS

Edited by:

Bin Cao,

China-Japan Friendship

Hospital, China

Reviewed by:

Anusak Kerdsin,

Kasetsart University, Thailand

Xiaojiong Jia,

Harvard Medical School,

United States

*Correspondence:

Xiaoqing Liu

Ixq1118@126.com

Yonghao Xu

amusing2000@163.com

tThese authors have contributed equally to this work

Specialty section:

This article was submitted to Infectious Diseases - Surveillance,

Prevention and Treatment, a section of the journal

Frontiers in Medicine

Received: 08 October 2021 Accepted: 23 December 2021

Published: 27 January 2022

Citation:

Wu Z, Zhang R, Liu D, Liu X, Zhang J, Zhang Z, Chen S, He W, Li Y, XU Y and Liu X (2022) Acute Respiratory

Distress Syndrome Caused by Human Adenovirus in Adults: A Prospective Observational Study in Guangdong,

China. Front. Med. 8:791163.

doi: 10.3389/fmed.2021.791163

\section{Acute Respiratory Distress Syndrome Caused by Human Adenovirus in Adults: A Prospective Observational Study in Guangdong, China}

\author{
Zhan Wu ${ }^{\dagger}$, Rong Zhang ${ }^{\dagger}$, Dongdong Liu ${ }^{\dagger}$, Xuesong Liu, Jierong Zhang, Zhihui Zhang, \\ Sibei Chen, Weiqun He, Yimin Li, Yonghao Xu* and Xiaoqing Liu*
}

Department of Critical Care Medicine, State Key Laboratory of Respiratory Diseases, National Clinical Research Center for Respiratory Disease, Guangzhou Institute of Respiratory Health, The First Affiliated Hospital of Guangzhou Medical University, Guangzhou, China

Background: Viral causes of acute respiratory distress syndrome (ARDS) are mostly limited to influenza. However, adenovirus has been emerging as a cause of ARDS with a high mortality rate and described in adults are rare.

Methods: We conducted a prospective, single-center observational study of viral pneumonia with ARDS and confirmed adenovirus-associated ARDS in adults at our quaternary referral institution between March 2019 and June 2020. We prospectively analyzed clinical characteristics, laboratory test results, radiological characteristics, viral load from nasopharyngeal swabs and endotracheal aspirates, treatments, and outcomes for the study participants.

Results: The study enrolled 143 ARDS patients, including 47 patients with viral pneumonia-related ARDS, among which there were 14 adenovirus-associated ARDS patients, which accounted for $29.79 \%$ of the viral pneumonia-related ARDS cases. Among the adenovirus-associated ARDS patients, $78.57 \%$ were men with a mean age of $54.93 \pm 19.04$ years, younger than that of the non-adenovirus associated ARDS patients. Adenovirus-associated ARDS patients had no specific clinical characteristics, but they presented with decrease in the number of CD3+CD4+ T cells and higher serum creatinine during the early stage. The viral load and the positivity rate in the lower respiratory tract were higher than that of the upper respiratory tract in the patients with adenovirus-associated ARDS. All patients required invasive mechanical ventilation treatment. The average time from shortness of breath to the application of invasive ventilation was $24 \mathrm{~h}$. Ten patients $(71.43 \%)$ complicated by acute kidney injury, while 13 patients $(71.43 \%)$ in the non-adenovirus associated ARDS group $(P=0.045)$. Additionally, $85.71 \%$ of the 14 adenovirus-associated ARDS patients survived. No significant differences were detected between the two groups regarding duration of ventilation, length of ICU stay and mortality.

Conclusion: Adenovirus infection is an important cause of virus-related ARDS. The positivity rate of adenovirus infection in lower respiratory tract secretions was higher than 
that in upper respiratory tract secretions in these patients. Age, lower CD3+CD4+ T cells, and high serum creatinine may be were associated with adenovirus induce ARDS in adults required mechanical ventilation. Early identification and intervention to prevent disease progression are essential for reducing the mortality rate in these patients.

Keywords: acute respiratory distress syndrome, adenovirus, severe pneumonia, infection, adults

\section{INTRODUCTION}

Acute respiratory distress syndrome (ARDS) is a rapid inflammatory lung injury with a high mortality rate that ranges from $30.0-46.0 \%(1-3)$. Infectious pneumonia is the most common disease leading to ARDS, with viral infection accounting for $\sim 22-40 \%$ of cases $(4,5)$. Influenza and rhinovirus have been most commonly detected in viral pneumonias followed by parainfluenza, adenovirus, respiratory syncytial virus, coronavirus, and human metapneumovirus $(4,6)$. Adenovirus have been recently found to be responsible for infection in ventilated or non-ventilated ARDS patients. However, the proportion of viral pneumonia leading to ARDS is unknown and the true impact of these viral infections on outcomes remains to be determined.

Some viral infections, such as influenza A H1N1, H5N1, and H7N9 and the coronaviruses severe acute respiratory syndrome (SARS), severe acute respiratory syndrome coronavirus-2 (SARSCoV-2), and Middle East Respiratory Syndrome (MERS), are associated with a high incidence of ARDS and increased mortality $(7,8)$. Adenovirus-associated ARDS has been reported in adult patients with rapid progression to multi-organ failure and death, which has raised concerns (9). However, there is limited information on adult adenovirus-associated ARDS and no consensus on its management (10).

Human adenoviruses (HAdVs) are non-enveloped DNA viruses that are associated with a wide range of clinical manifestations (11). Adenovirus infection that causes severe fatal pneumonia has been well described in infants and children, but reports in adults are rare (12). HAdV-associated ARDS infection is a devastating disease with rapid progression to multi-organ failure and death, and it can be fulminant with a mortality rate of $\sim 40 \%$ in patients who require mechanical ventilation (13). However, ARDS caused by HAdV in adults required mechanical ventilation was rarely reported. Here, we performed a prospective, observational study to evaluate the proportion of viral pneumonia leading to ARDS in adult patients, we also analyzed to compare sequential viral load test results on nasopharyngeal swabs and endotracheal aspirates, and finally set a comparison group to reveal the risk factors of adenovirusassociated ARDS requiring mechanical ventilation.

\section{METHODS}

\section{Study Design and Population}

We performed a prospective, single-center observational study in adult patients with viral pneumonia with ARDS who were admitted to our respiratory intensive care unit (ICU) between March 2019 and June 2020. The study was approved by the Ethics Committee of the First Affiliated Hospital of Guangzhou Medical
University. All patients provided written informed consent for their data to be used for research.

Patients were included if they met the following inclusion criteria: age $\geq 18$ years, patients receiving invasive mechanical ventilation with a diagnosis of ARDS $<24 \mathrm{~h}$ before enrollment in the trial. ARDS was diagnosed in accordance with the Berlin definition (2), as follows: (1) developing within 1 week of a known clinical insult or new or worsening respiratory symptoms; (2) bilateral opacities not fully explained by effusions, lobar and/or lung collapse, or nodules; (3) respiratory failure not fully explained by cardiac failure or fluid overload; (4) partial oxygen pressure/fraction of inspired oxygen $\left(\mathrm{PaO}_{2} / \mathrm{FiO}_{2}\right)$ $\leq 300 \mathrm{mmHg}$ with positive end-expiratory pressure (PEEP) $\geq 5$ $\mathrm{cmH}_{2} \mathrm{O}$; and (5) a chest radiograph with three or four quadrants with opacities. Patients with HAdV or other virus's infection confirmed by chest X-ray, a positive RT-PCR finding from an airway specimen, requiring invasive mechanical ventilation were included in the analysis. The exclusion criteria were age younger than 18 years; pregnant women, or inability or unwillingness to provide informed consent.

\section{Clinical Data Collection}

A standardized electronic case report form was used to prospectively collect the study data. The following data were obtained: age, sex, body mass index (BMI), preexisting comorbidities, Acute Physiology and Chronic Health Evaluation II (APACHEII) score, daily Sequential Organ Failure Assessment (SOFA) score, and clinical pulmonary infection score (CPIS). Clinical symptoms (fever, cough, sputum, and dyspnea), signs (body temperature, heart rate, respiratory frequency, and blood pressure), and laboratory data (procalcitonin, white blood cell count, lymphocyte, platelet count, and creatinine level), microbiological findings, and images of the lung (chest X-ray and computed tomography) were also collected. Additionally, complications, treatments, respiratory support, and clinical outcomes were recorded.

\section{Molecular Assay for Respiratory Virus Detection}

Nasopharyngeal swabs and endotracheal aspirates (ETA) were collected at admission and during hospitalization and stored at $-80^{\circ} \mathrm{C}$ until testing. All nasopharyngeal swabs and ETA samples were analyzed using multiplex quantitative polymerase chain reaction (Q-PCR) technology. Viral nucleic acid was extracted from the samples using the QIAamp MiniElute Virus Spin kit (QIAGEN, Germany) following the manufacturer's instructions. This assay tested for 24 respiratory viruses (adenovirus; influenza virus type A H1N1, H3N2, H1N1 2009, H5N1, and H7N9; influenza virus type $B$; respiratory syncytial virus types $A$ and 


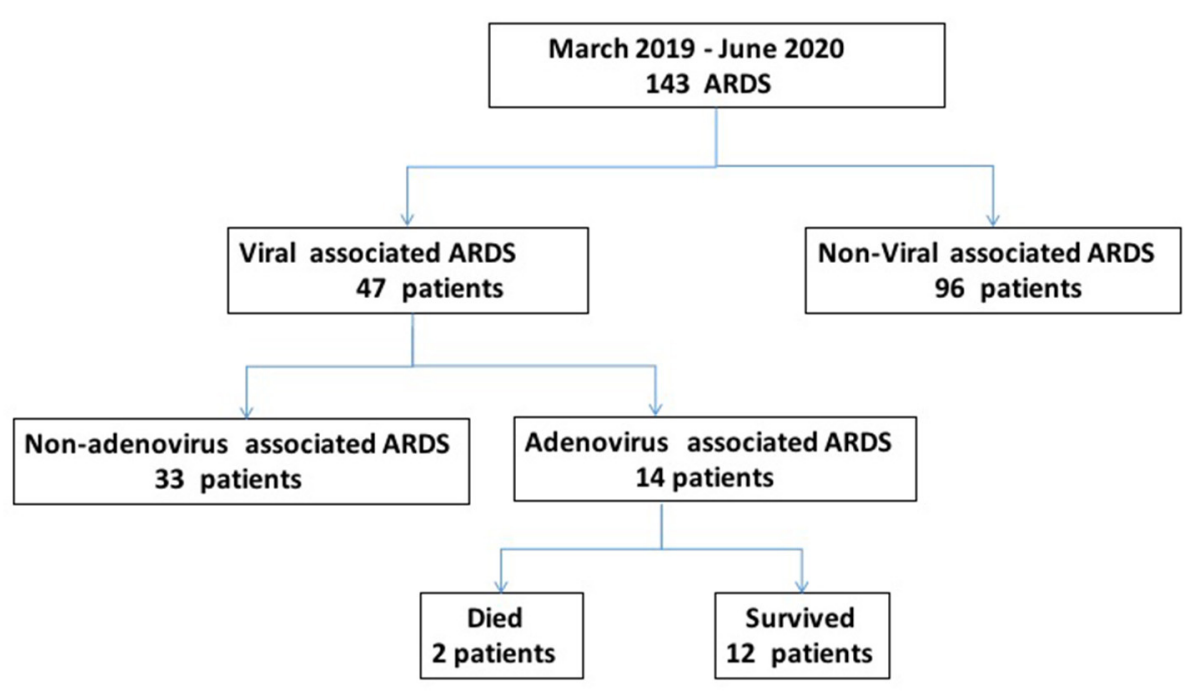

FIGURE 1 | Flowchart of patient groups in the study. ARDS, acute respiratory distress syndrome.

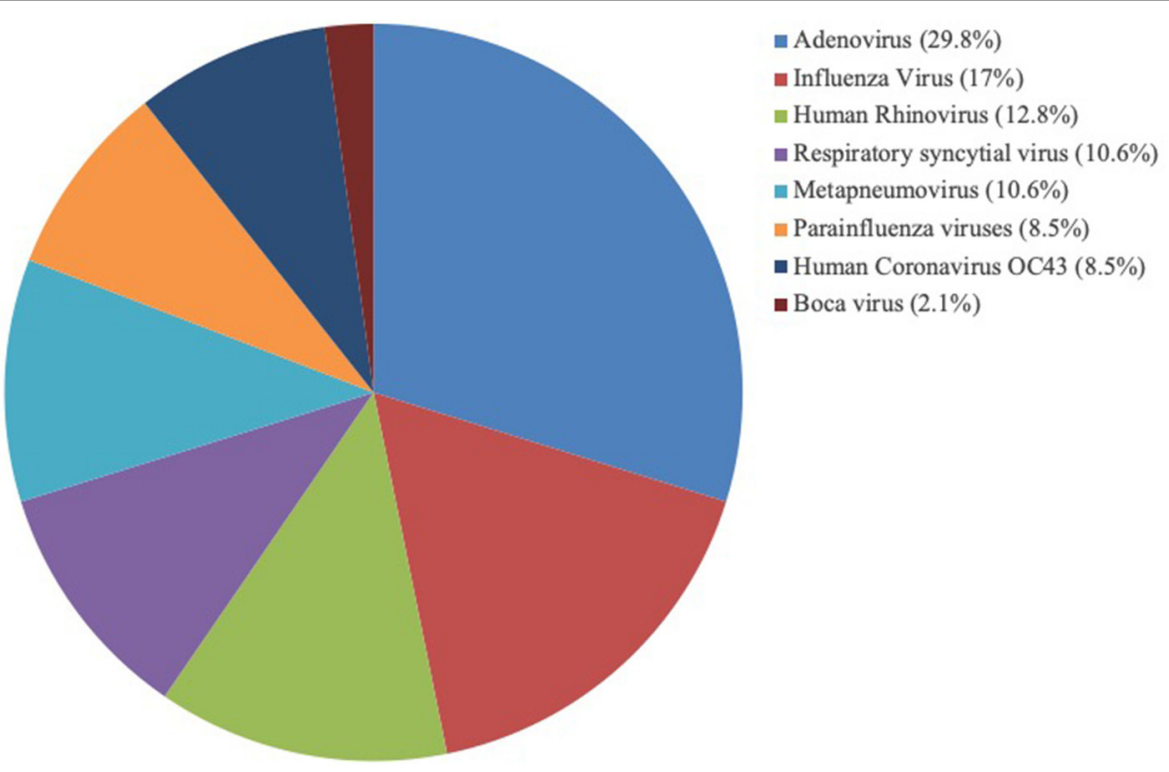

FIGURE 2 | Distribution of viruses detected in 47 ARDS patients.

B; parainfluenza viruses type 1, 2, 3, and 4; metapneumovirus; coronavirus OC43, 229E, NL63, HKU1, SARS, and MERS; rhinovirus; bocavirus; Nipah virus; and circovirus).

The viral load was indicated as the cycle threshold $(\mathrm{Ct})$ value. $\mathrm{Ct}$ values were used to analyze each patient's viral load. Ct values were inversely correlated to the quantity of RNA target present in the specimen. A Ct value of $<40$ was defined as positive for the viruses and $>40$ was defined as a negative result. Samples with a $\mathrm{Ct}$ value between 37 and 40 were retested at least twice.

\section{Statistical Analysis}

Measurement variables were summarized using the median (interquartile range) or the mean and range, and enumeration variables were presented using the frequency and percentage.
Differences between groups were assessed with the Fisher exact test for categorical variables and the Mann-Whitney $U$-test for continuous variables, and $p<0.05$ was considered to be significant. Statistical analysis was performed using GraphPad Prism 7.0.

\section{RESULTS}

The study included 143 patients, including 47 patients $(32.87 \%)$ with viral pneumonia-related ARDS and 14 adenoviruspositive ARDS patients, which accounted for $29.79 \%$ of viral pneumonia-related ARDS (Figure 1). As shown in Figure 2, among the non-adenovirus associated ARDS patients, the most common finding was Influenza virus, detected in $17 \%$ of the 
samples, followed by human rhinovirus (12.8\%), respiratory syncytial virus (10.6\%), and human metapneumovirus (10.6\%), parainfluenza viruses $(8.5 \%)$, human coronavirus OC43 (8.5\%), boca virus $(2.1 \%)$.

The general condition and clinical characteristics of the patients with adenovirus-associated ARDS and non-adenovirus associated ARDS are shown in Table 1. The mean age was 54.93 \pm 19.04 years among the adenovirus-associated ARDS patients, younger than that of the non-adenovirus associated ARDS patients. Among the adenovirus-associated ARDS patients, $78.57 \%$ were males, $50 \%$ were smokers, and $71.43 \%$ had high nutritional risk (Table 1). In addition, $28.57 \%$ of the adenovirusassociated patients had concurrent lung diseases, and $28.57 \%$ of the patients also had an immunodeficiency. All patients had cough, sputum expectoration, and shortness of breath, and $92.86 \%$ of them had a fever. Most patients presented with a lowgrade fever. As shown in Table 2, the $\mathrm{PaO}_{2} / \mathrm{FiO}_{2}$ for $64.29 \%$ of the adenovirus-associated patients was between 150 and $200 \mathrm{mmHg}$. Most of the patients had normal blood $\mathrm{CO}_{2}$, manifesting as type I respiratory failure. The average time from the onset of shortness of breath to tracheal intubation was $24 \mathrm{~h}$. Hypoproteinemia occurred in $85.71 \%$ of the adenovirus-associated ARDS patients, and abnormal coagulation function occurred in $>60 \%$ of the patients. At the time of enrollment, $64.29 \%$ of the patients had a low total number of white blood cells, and more than $70 \%$ of the patients had a decrease in the total number and percentage of lymphocytes. Additionally, $85.71 \%$ of the adenovirus-associated patients had a significant decrease in the number of CD3+CD4+ $\mathrm{T}$ cells and higher serum creatinine during the early stage compared with the non-adenovirus associated ARDS patients. Most of the adenovirus-associated patients had diffuse infiltrates in both lungs. Three $(21.43 \%)$ of the patients had interstitial abnormalities. Five patients $(35.71 \%)$ had pleural effusions. Only a few patients showed ground-glass opacity (Figure 3).

Within $48 \mathrm{~h}$ of enrollment, paired upper respiratory tract (URT) samples (nasopharyngeal swab) and lower respiratory tract (LRT) samples (sputum and bronchoalveolar lavage) were collected from the adenovirus-associated ARDS patients to detect the virus using multiplex Q-PCR. As shown in Table 3, the LRT samples showed a significantly higher positive rate $(100 \%, 14 / 14)$ than URT samples $(64.29 \%, 9 / 14)$ for detecting respiratory viruses $(p<0.001)$, and LRT samples had higher levels of detection using a $\mathrm{Ct}$ value of 31.66 (20.56-34.78) compared with URT sampling using a Ct value of 39.14 (27.95-40) (Figure 4). On average, URT sites cleared faster than LRT sites (Figure 5). Detection of other viruses, fungi, and bacteria from the sputum collected from patients within $48 \mathrm{~h}$ of enrollment showed that four patients (28.57\%) also had another viral infection, and among these patients, the most common virus was the influenza virus that accounted for $14.29 \%$ of viral infections. Nine patients (64.29\%) had positive bacterial cultures in the sputum, with the highest positivity rate for Acinetobacter baumannii, which was detected in six patients (42.86\%). All patients had a negative fungal culture from the sputum, blood, and bronchoalveolar lavage fluid in (1-3)- $\beta$-D-glucan (G) and galactomannan (GM) tests.
TABLE 1 | Demographic data and clinical characteristics of the patients with adenovirus-associated ARDS and non-adenovirus associated ARDS.

\begin{tabular}{|c|c|c|c|}
\hline & $\begin{array}{l}\text { Adenovirus } \\
\text { associated } \\
\text { ARDS }(n=14)\end{array}$ & $\begin{array}{l}\text { Non-adenovirus } \\
\text { associated ARDS } \\
(n=33)\end{array}$ & $P$-value \\
\hline Sex(male) & $11(78.57 \%)$ & 23(69.70\%) & 0.79 \\
\hline Age(years) & $54.93 \pm 19.04$ & $64.40 \pm 11.20$ & 0.05 \\
\hline BMI $\left(\mathrm{kg} / \mathrm{m}^{2}\right)$ & $20.30 \pm 3.03$ & $20.76 \pm 6.83$ & 0.81 \\
\hline APACHEll score & $19.14 \pm 7.35$ & $21.06 \pm 8.37$ & 0.46 \\
\hline SOFA score & $10.50 \pm 4.09$ & $8.94 \pm 3.77$ & 0.21 \\
\hline CPIS score & $6.50 \pm 1.15$ & $6.06 \pm 0.93$ & 0.60 \\
\hline Smoking & $7(50 \%)$ & 11(33.33\%) & 0.28 \\
\hline High nutritional risk & $9(64.29 \%)$ & $25(75.76 \%)$ & 0.65 \\
\hline Underlying lung disease & $4(28.57 \%)$ & $12(36.36 \%)$ & 0.86 \\
\hline COPD & $2(14.28 \%)$ & $11(33.33 \%)$ & 0.33 \\
\hline Bronchiectasis & $1(7.14 \%)$ & $1(3.03 \%)$ & 0.52 \\
\hline Interstitial lung disease & $1(7.14 \%)$ & $1(3.03 \%)$ & 0.52 \\
\hline Immunodeficiency & $4(28.57 \%)$ & $8(24.24 \%)$ & $>0.99$ \\
\hline \multicolumn{4}{|l|}{ Symptoms } \\
\hline Fever & 13(92.86\%) & 23(69.70\%) & 0.18 \\
\hline Cough & $14(100.00 \%)$ & $33(100 \%)$ & \\
\hline Shortness of breath & $14(100.00 \%)$ & $33(100 \%)$ & \\
\hline \multicolumn{4}{|l|}{ Temperature $\left({ }^{\circ} \mathrm{C}\right)$} \\
\hline $36.0-37.2$ & $7(50 \%)$ & 23(69.70\%) & 0.20 \\
\hline 37.3-38.0 & $5(35.71 \%)$ & $4(12.12 \%)$ & 0.14 \\
\hline 38.1-39.0 & $1(7.14 \%)$ & $5(15.15 \%)$ & 0.45 \\
\hline $39.1-41$ & $1(7.14 \%)$ & $1(3.03 \%)$ & 0.52 \\
\hline Heart rate(bpm) & $98.36 \pm 24.11$ & $101.58 \pm 26.00$ & 0.70 \\
\hline Respiratory rate(bpm) & $22.93 \pm 6.96$ & $23.36 \pm 7.23$ & 0.85 \\
\hline MAP (mmHg) & $75.86 \pm 12.27$ & $77.86 \pm 10.35$ & 0.74 \\
\hline \multicolumn{4}{|l|}{ Co-infection } \\
\hline Other viruses & $4(28.57 \%)$ & 0 & \\
\hline Influenza virus & $2(14.29 \%)$ & 0 & \\
\hline Human Coronavirus OC43 & $1(7.14 \%)$ & 0 & \\
\hline Human rhinovirus & $1(7.14 \%)$ & 0 & \\
\hline Bacteria & $10(71.43 \%)$ & 23(69.70\%) & $>0.99$ \\
\hline Acinetobacter baumannii & $6(42.86 \%)$ & $8(24.24 \%)$ & 0.35 \\
\hline Stenotrophomonas Maltophilia & $3(21.43 \%)$ & $7(21.21 \%)$ & $>0.99$ \\
\hline Klebsiella pneunoniae & $2(14.29 \%)$ & 3(9.09\%) & $>0.99$ \\
\hline Other bacteria & $4(28.57 \%)$ & $7(21.21 \%)$ & 0.87 \\
\hline
\end{tabular}

All patients received antiviral therapy, including ganciclovir ( $5 \mathrm{mg} / \mathrm{kg}$ intravenously every $12 \mathrm{~h}$ ) and oseltamivir. Considering that bacterial coinfection may combine with a severe viral infection, broad-spectrum intravenous antibiotics were administered to all patients. Additionally, $78.57 \%$ of the patients received immunoglobulin. All patients required invasive ventilation, the median duration of invasive mechanical ventilation was 22 days (14-75 days), compared with 22 days (range, 10-34 days) in the non-adenovirus associated ARDS group. Ten patients (71.43\%) complicated by acute kidney injury, while 13 patients $(71.43 \%)$ in the non-adenovirus associated ARDS group $(P=0.045)$. Three patients $(21.43 \%)$ required extracorporeal membrane oxygenation (ECMO), with 
TABLE 2 | Laboratory findings and radiographic results for patients with adenovirus-associated ARDS.

\begin{tabular}{|c|c|c|c|}
\hline Characteristic & $\begin{array}{l}\text { Adenovirus } \\
\text { associated } \\
\text { ARDS }(n=14)\end{array}$ & $\begin{array}{l}\text { Non-adenovirus } \\
\text { associated ARDS } \\
(n=33)\end{array}$ & $P$-value \\
\hline White blood cell count, $\times 10^{9} / L$ & $12.66 \pm 6.40$ & $11.95 \pm 6.67$ & 0.84 \\
\hline$<4$ & 0 & 3(9.09\%) & 0.24 \\
\hline$>10$ & 9(64.29\%) & $14(42.42 \%)$ & 0.17 \\
\hline Platelet count, $\times 10^{9} / \mathrm{L}$ & $\begin{array}{c}165.43 \pm \\
103.26\end{array}$ & $168.24 \pm 75.45$ & 0.92 \\
\hline$<100$ & $5(35.71 \%)$ & $6(18.18 \%)$ & 0.36 \\
\hline Lymphocyte, $\times 10^{9} / \mathrm{L}$ & $0.64 \pm 0.49$ & $0.54 \pm 0.24$ & 0.59 \\
\hline$<0.9$ & 10(71.43\%) & 28(84.80\%) & 0.51 \\
\hline CD3+CD45+ T, Cell/Ul & $\begin{array}{c}278.00(171.50- \\
634.00)\end{array}$ & $\begin{array}{c}343.00(138.00- \\
665.00)\end{array}$ & 0.67 \\
\hline$<955$ & $12(85.71 \%)$ & $24(72.73 \%)$ & 0.56 \\
\hline CD3+CD4+ T, Cell/Ul & $\begin{array}{c}148.00(106.00- \\
365.00)\end{array}$ & $\begin{array}{c}237.00(101.00- \\
596.00)\end{array}$ & 0.08 \\
\hline$<550$ & $12(85.71 \%)$ & $18(54.55 \%)$ & 0.041 \\
\hline CD3+CD8+ T, Cell/UI & $\begin{array}{c}120.00(57.50- \\
282.00)\end{array}$ & $\begin{array}{c}107.00(62.00- \\
259.00)\end{array}$ & 0.91 \\
\hline$<320$ & $11(78.57 \%)$ & 22(66.66\%) & 0.64 \\
\hline Interleukin-6, pg/ml & $\begin{array}{c}39.37(12.53- \\
143.06)\end{array}$ & $22.93(12.40-140.14)$ & 0.84 \\
\hline$>5.3$ & 12(85.71\%) & 22(66.66\%) & 0.33 \\
\hline Procalcitonin, ng/ml & 0.38(1.90-4.66) & 0.29(1.69-5.43) & 0.98 \\
\hline \multicolumn{4}{|l|}{ Arterial blood gas analysis: } \\
\hline $\mathrm{pH}$ & $7.37 \pm 0.09$ & $7.37 \pm 0.12$ & 0.90 \\
\hline $\mathrm{pCO}_{2}, \mathrm{mmHg}$ & $44.12 \pm 6.83$ & $47.09 \pm 10.26$ & 0.33 \\
\hline $\mathrm{P} / \mathrm{F}$ & $168.59 \pm 41.77$ & $153.13 \pm 53.77$ & 0.34 \\
\hline$<150$ & $5(35.71 \%)$ & $20(60.61 . \%)$ & 0.12 \\
\hline $150-200$ & $9(64.29 \%)$ & $8(24.24 \%)$ & 0.01 \\
\hline Albumin, $g / L$ & $30.96 \pm 10.14$ & $31.21 \pm 6.89$ & 0.92 \\
\hline$<35$ & $12(85.71 \%)$ & $28(84.85 \%)$ & $>0.99$ \\
\hline Prothrombin time, s & $16.06 \pm 2.73$ & $16.24 \pm 2.15$ & 0.80 \\
\hline$>14.5$ & $10(71.43 \%)$ & 29(87.88.\%) & 0.34 \\
\hline $\begin{array}{l}\text { Activated partial thromboplastin } \\
\text { time,s }\end{array}$ & $51.23 \pm 28.21$ & $48.98 \pm 20.04$ & 0.76 \\
\hline$>42.8$ & $9(64.29 \%)$ & $16(48.48 \%)$ & 0.32 \\
\hline Creatinine, umol/L & $\begin{array}{c}162.55(87.50- \\
282.50)\end{array}$ & 73.0(60.0-117.30) & 0.047 \\
\hline$>133$ & $9(64.29 \%)$ & $7(21.21 \%)$ & 0.01 \\
\hline \multicolumn{4}{|c|}{ Abnormalities on chest radiograph } \\
\hline Ground-glass opacity & $2(14.29 \%)$ & $1(3.03 \%)$ & 0.43 \\
\hline Bilateral patchy shadowing & $14(100 \%)$ & 29(87.88\%) & 0.17 \\
\hline Interstitial abnormalities & $3(21.43 \%)$ & $4(12.12 \%)$ & 0.71 \\
\hline Pleural effusions & $5(35.71 \%)$ & $6(18.18 \%)$ & 0.36 \\
\hline
\end{tabular}

a treatment duration of 7 to 12 days, and one patient $(7.14 \%)$ underwent prone position ventilation. The average length of ICU stay was 26.50 days (IQR, 15-75 days), and the median length of hospital stay was 37.5 days (IQR, 29.75-81.00 days). Among the 14 patients in this study, there were two deaths, with a mortality rate of $14.29 \%$ during the study period (Table 4 ). No significant

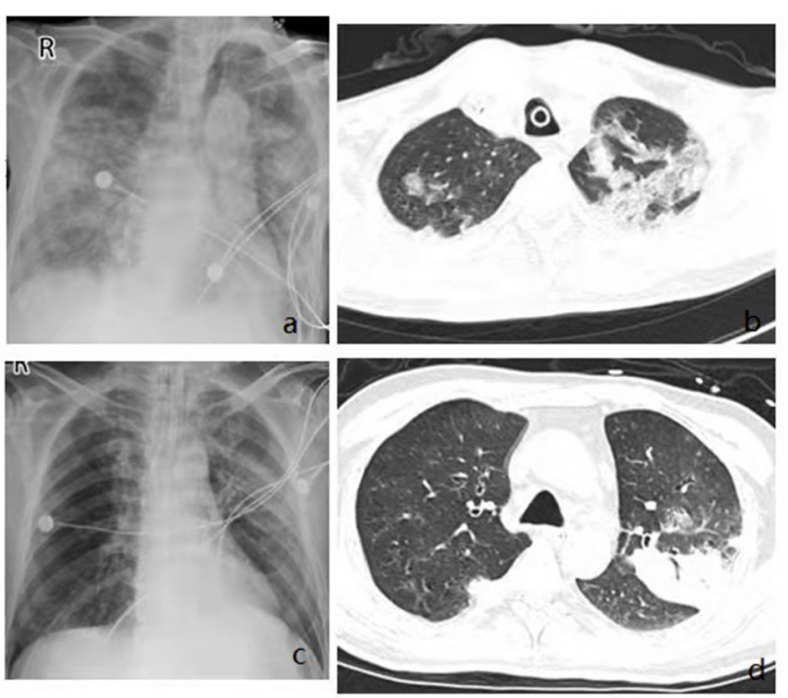

FIGURE 3 | Imaging findings showing diffuse multifocal or diffuse opacity. Chest $x$ rays from admission showing bilateral diffuse infiltrates $(\mathbf{a}, \mathbf{c})$, Chest CT from admission showing multifocal consolidation and groundglass opacity in both lungs $(\mathbf{b}, \mathbf{d})$.

TABLE 3 | Comparison of multiplex Q-PCR results between URT and LRT samples.

\begin{tabular}{llll}
\hline & URT & LRT & $P$-value \\
\hline Detected $n(\%)$ & $9(64.29 \%)$ & $14(100 \%)$ & $<0.001$ \\
Undetected $n(\%)$ & $5(35.71 \%)$ & 0 & $<0.001$ \\
Time of virus clearance(days) & $15.16(4-37)$ & $24.17(6-42)$ & $<0.001$
\end{tabular}

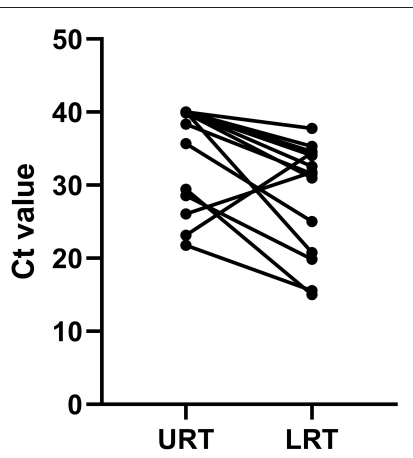

FIGURE 4 | Comparison of the Ct value between URT and LRT samples upon admission to the ICU. URT, upper respiratory tract; LRT, lower respiratory tract; ICU, intensive care unit; $\mathrm{Ct}$, cycle threshold.

differences were detected between the two groups regarding with the mortality.

\section{DISCUSSION}

The number of ARDS cases caused by viral pneumonia is increasing, resulting in a high mortality rate (14). Previous 


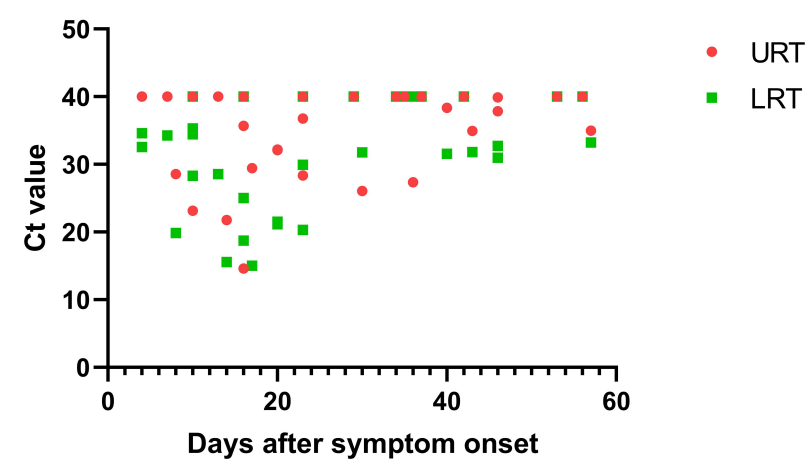

FIGURE 5 | The relationship between Ct value and days after symptom onset in the URT and LRT samples. URT, upper respiratory tract; LRT, lower respiratory tract; ICU, intensive care unit; Ct, cycle threshold.

TABLE 4 | Complications, treatments, and clinical outcomes in patients with adenovirus-associated ARDS.

\begin{tabular}{|c|c|c|c|}
\hline Characteristic & $\begin{array}{l}\text { Adenovirus } \\
\text { associated ARDS } \\
(n=14)\end{array}$ & $\begin{array}{l}\text { Non-adenovirus } \\
\text { associated ARDS } \\
(n=33)\end{array}$ & $P$-value \\
\hline \multicolumn{4}{|l|}{ Complications } \\
\hline AKI & $10(71.43 \%)$ & $13(39.39 \%)$ & 0.045 \\
\hline Sepsis shock & $4(28.57 \%)$ & $12(36.36 \%)$ & 0.86 \\
\hline Pneumothorax & $2(14.29 \%)$ & 0 & 0.02 \\
\hline \multicolumn{4}{|l|}{ Treatments } \\
\hline CRRT & $6(42.86 \%)$ & $10(30.30 \%)$ & 0.62 \\
\hline ECMO & $3(21.43 \%)$ & $2(6.06 \%)$ & 0.30 \\
\hline Prone position ventilation & $1(7.14 \%)$ & $2(6.06 \%)$ & 0.89 \\
\hline Vasoactive & $11(78.57 \%)$ & $25(75.76 \%)$ & $>0.99$ \\
\hline Muscle relaxants & $11(78.57 \%)$ & $20(60.61 \%)$ & 0.39 \\
\hline Intravenous immune globulin & $6(42.86 \%)$ & $10(30.30 \%)$ & 0.62 \\
\hline \multicolumn{4}{|l|}{ Anti-viral agents } \\
\hline Oseltamivir & $7(50.00 \%)$ & 11(33.33\%) & 0.28 \\
\hline Ganciclovir & $11(78.57 \%)$ & $9(27.27 \%)$ & 0.001 \\
\hline \multicolumn{4}{|l|}{ Clinical outcomes } \\
\hline Duration of dyspnea to IMV & $1.00(1.00-2.00)$ & $3.00(2.00-5.00)$ & $>0.99$ \\
\hline Duration of IMV (days) & $22.00(14.00-75.25)$ & 22.00(10.00-34.00) & 0.41 \\
\hline Length of ICU stay(days) & $26.50(15.75-75.25)$ & $27.00(11.50-41.50)$ & 0.52 \\
\hline Length of hospital stay(days) & $37.50(29.75-81.00)$ & $46.00(27.50-57.50)$ & 0.89 \\
\hline Death & $2(14.29 \%)$ & $4(12.12 \%)$ & $>0.99$ \\
\hline
\end{tabular}

studies have mostly focused on influenza viruses, such as H1N1 and H7N9 $(15,16)$. Cases of adenovirus-associated ARDS have gradually been increasing, which may be due to recent developments in molecular diagnostic technology (17). However, there is limited data about the viral etiology of ARDS in patients who required mechanical ventilation. Zhou et al. reported that the incidence of adenovirus pneumonia ranks third among viral pneumonia in adults in China (6). They have also found that among virus-related ARDS patients with $\mathrm{PO}_{2} / \mathrm{FiO}_{2}$ ratio < $200 \mathrm{mmHg}$, HAdV infection was the most frequently detected virus (6). Our study showed that in all ARDS patients, virusrelated ARDS accounted for $32.87 \%$ of infections. Among these infections, adenovirus-associated ARDS accounted for 9.79\% of all ARDS patients and $29.79 \%$ of virus-related ARDS patients. The prevalence of respiratory viruses varies in different countries and different populations $(4-6,13)$. To the best of our knowledge, this is the first study about the viral etiology in ARDS patients who required mechanical ventilation in China.

There are few studies on adenovirus-associated ARDS in adults who required mechanical ventilation (18-20). In 2000, two non-immunocompromised soldiers became infected with adenovirus, which resulted in ARDS (21). In 2006, there was an outbreak of adenovirus pneumonia caused by HAdV-B11 in the USA; 140 people were diagnosed with HAdV infection, and 24 patients who were diagnosed with ARDS were admitted to the ICU (22). Some studies have proposed that severe adenovirus infection is likely to occur in children and immunocompromised adults, such as HIV patients, and patients after transplantation $(23,24)$. In this study, most of the adenovirus-associated ARDS patients, with a mean age of 54 years, had no underlying diseases. Among these patients, $78.57 \%$ of them were men, and $50 \%$ of the patients were smokers, suggesting that severe adenovirus pneumonia in non-immunocompromised adults was likely to occur in middle-aged men. Additionally, $85.71 \%$ of the patients had a significant decrease in the number of $\mathrm{CD} 3+\mathrm{CD} 4+\mathrm{T}$ cells during the early stage, which suggests that adenovirus infection may cause immune system dysregulation.

Delayed clearance of respiratory adenovirus infection leads to a worse prognosis in these patients, and monitoring the viral load may help to predict the disease severity and the patients' prognosis $(25,26)$. Rapid identification of adenovirus viral infection is critical to reduce the overall costs of patient management. Multiplex Q-PCR is of great value in the early diagnosis of virus infection because of its high sensitivity (27, 28). However, viral testing of URT and LRT samples may yield different results (29). Currently, few studies have been published that compare the diagnostic yields of URT and LRT samples to detect adenovirus. In our study, the detection rates of adenovirus from LRT and URT samples were 100 and $64.29 \%$, respectively. Similarly, a European study reported that the overall virus positivity rate of URT was lower than that of the LRT specimens (24.5 vs. $44.2 \%$ ) (30). In this study, the percentage of positive specimens was higher in LRT than in URT specimens. On average, URT specimens cleared faster than LRT specimens, suggesting that traditional nasopharyngeal diagnostic techniques can miss cases of severe adenovirus infection. This suggests that LRT specimens are more reliable for diagnosing severe adenovirus infection, especially in patients with pneumonia that occurs several days after the infection onset when the frequency of virus detection in the URT has already decreased.

Several studies have shown that the mortality of severe adenovirus-associated ARDS can be as high as $26.7-80 \%$ in adults $(10,31)$. In our study, among the 14 patients with adenovirus-associated ARDS, there were only two deaths, and the mortality rate was $14.29 \%$. Compared with previous studies, the mortality rate of adenovirus-associated ARDS in this study was relatively low, and this may have several explanations. First, rapid identification of adenovirus viral infection and early intervention are important to reduce the overall mortality rate. 
In this study, the time from onset to intubation was relatively short. In addition, the $\mathrm{PO}_{2} / \mathrm{FiO}_{2}$ ratio for most adenovirusassociated ARDS patients was $>150$ in this study, while most other studies showed that the $\mathrm{PO}_{2} / \mathrm{FiO}_{2}$ ratio in patients with severe pneumonia was $<150(6,23,31)$. Considering that the condition of severe adenovirus pneumonia patients was more advanced, the above results suggested that early intervention in adenovirus pneumonia-related ARDS helped to improve the patients' prognosis. Second, establishing organ support, such as application of early renal replacement treatment and ECMO are important. Adenovirus-associated ARDS completely resolved in three patients who were supported by ECMO in this study, suggesting that early application of ECMO improved the prognosis of patients with adenovirus-associated ARDS. Finally, timely initiation of antiviral therapy is very important to improve patient outcome. Currently, antiviral therapies for adenovirus infection remain controversial. No specific and effective antiviral drug is available for adenovirus infection (32). Some studies have shown that cidofovir antiviral therapy in severe adenovirus pneumonia improves the clinical prognosis (33). However, clinical application of cidofovir is limited due to its toxic side effects and low-quality evidence. Other case reports have also shown that ribavirin can be used to treat adenovirus infection $(34,35)$. Ganciclovir has been shown to be effective for treating adenovirus infection in animal experiments (36). In our study, 11 patients $(78.57 \%)$ received antiviral therapy with ganciclovir and immunoglobulin therapy after confirming adenovirus infection, which might be the reason for the lower mortality.

This study prospectively observed the viral etiology of ventilated ARDS patients, especially for patients where adenovirus was associated with ARDS. We compared the adenovirus detection rate and adenovirus load in different respiratory tract specimens, which were also a highlight of the study. This study also has several limitations. It was a single-center study with a relatively small number of patients enrolled. In addition, this study used multiplex Q-PCR to detect multiple respiratory viruses at the same time without genotyping the adenovirus.

\section{CONCLUSIONS}

Our findings indicated that adenovirus infection was an important cause of viral-related ARDS. The detection rate of

\section{REFERENCES}

1. Thompson BT, Chambers RC, Liu KD. Acute respiratory distress syndrome. N Engl J Med. (2017) 377:562-72. doi: 10.1056/NEJMra1608077

2. Confalonieri M, Salton F, Fabiano F. Acute respiratory distress syndrome. Eur Respir Rev. (2017) 26:160116. doi: 10.1183/16000617.0116-2016

3. Chiumello D, Brochard L, Marini JJ, Slutsky AS, Mancebo J, Ranieri VM, et al. Respiratory support in patients with acute respiratory distress syndrome: an expert opinion. Crit Care. (2017) 21:240. doi: 10.1186/s13054-017-1820-0

4. Jain S, Self WH, Wunderink RG, Fakhran S, Balk R, Bramley AM, et al. Community-acquired pneumonia requiring hospitalization among U.S. adults. N Engl J Med. (2015) 373:415-27. doi: 10.1056/NEJMoa1500245 adenovirus from the LRT was higher than that from the URT. Age, lower $\mathrm{CD} 3+\mathrm{CD} 4+\mathrm{T}$ cells, and high serum creatinine may be were associated with adenovirus induce ARDS in adults required mechanical ventilation. Early identification and effective intervention to prevent disease progression are essential for reducing the mortality rate in these patients.

\section{DATA AVAILABILITY STATEMENT}

The original contributions presented in the study are included in the article/supplementary material, further inquiries can be directed to the corresponding author/s.

\section{ETHICS STATEMENT}

The studies involving human participants were reviewed and approved by the Ethics Committee of the First Affiliated Hospital of Guangzhou Medical University (2019-19). The patients/participants provided their written informed consent to participate in this study. Written informed consent was obtained from the individual(s) for the publication of any potentially identifiable images or data included in this article.

\section{AUTHOR CONTRIBUTIONS}

XiL: study conception and design. ZW, RZ, DL, XuL, SC, YX, JZ, and ZZ: data collection. ZW, RZ, WH, XiL, and YL: analysis and interpretation. RZ and ZW: writing the manuscript. All authors have read and approved the final manuscript.

\section{FUNDING}

This study was supported by National Natural Science Foundation of China (Nos. 82070084 to XiL and 81700080 to RZ) and National Science and Technology Major Project (No. 2017ZX10204401003).

\section{ACKNOWLEDGMENTS}

We thank Jodi Smith, from Liwen Bianji (Edanz) (www.liwenbianji.cn/ac) for editing the English text of a draft of this manuscript.
5. Kalil AC, Thomas PG. Influenza virus-related critical illness: pathophysiology and epidemiology. Crit Care. (2019) 23:258. doi: 10.1186/s13054-019-2539-X

6. Zhou F, Wang Y, Liu Y, Liu X, Gu L, Zhang X, et al. Disease severity and clinical outcomes of community-acquired pneumonia caused by non-influenza respiratory viruses in adults: a multicentre prospective registry study from the CAP-China Network. Eur Respir J. (2019) 54:1802406. doi: 10.1183/13993003.02406-2018

7. Li X, Ma X. Acute respiratory failure in COVID-19: is it "typical" ARDS? Crit Care. (2020) 24:198. doi: 10.1186/s13054-020-02911-9

8. Herold S, Becker C, Ridge KM, Budinger GR. Influenza virus-induced lung injury: pathogenesis and implications for treatment. Eur Respir J. (2015) 45:1463-78. doi: 10.1183/09031936.00186214 
9. Cao B, Huang GH, Pu ZH, Qu JX, Yu XM, Zhu Z, et al. Emergence of community-acquired adenovirus type 55 as a cause of community-onset pneumonia. Chest. (2014) 145:79-86. doi: 10.1378/chest.13-1186

10. Sun B, He H, Wang Z, Qu J, Li X, Ban C, et al. Emergent severe acute respiratory distress syndrome caused by adenovirus type 55 in immunocompetent adults in 2013: a prospective observational study. Crit Care. (2014) 18:456. doi: 10.1186/s13054-014-0456-6

11. Binder AM, Biggs HM, Haynes AK, Chommanard C, Lu X, Erdman DD, et al. Human adenovirus surveillance - United States, 2003-2016. MMWR Morb Mortal Wkly Rep. (2017) 66:1039-42. doi: 10.15585/mmwr.mm6639a2

12. Wong-Chew RM, García-León ML, Noyola DE, Perez GL, Gaitan MJ, Vilaseñor-Sierra A, et al. Respiratory viruses detected in Mexican children younger than 5 years old with community-acquired pneumonia: a national multicenter study. Int J Infect Dis. (2017) 62:32-8. doi: 10.1016/j.ijid.2017.06.020

13. Vashisht R, Mirzai S, Koval C, Duggal A. Adenovirus-associated acute respiratory distress syndrome: need for a protocol-based approach. Indian J Crit Care Med. (2020) 24:367-8. doi: 10.5005/jp-journals-10071-23428

14. Matthay MA, Aldrich JM, Gotts JE. Treatment for severe acute respiratory distress syndrome from COVID-19. Lancet Respir Med. (2020) 8:4334. doi: 10.1016/S2213-2600(20)30127-2

15. Chen J, Hu C, Chen L, Tang L, Zhu Y, Xu X, et al. Clinical study of mesenchymal stem cell treatment for acute respiratory distress syndrome induced by epidemic influenza A (H7N9) infection: a hint for COVID-19 treatment. Engineering. (2020) 6:1153-61. doi: 10.1016/j.eng.2020.02.006

16. Tang X, Du RH, Wang R, Cao TZ, Guan LL, Yang CQ, et al. Comparison of hospitalized patients with ARDS caused by COVID-19 and H1N1. Chest. (2020) 158:195-205. doi: 10.1016/j.chest.2020.03.032

17. Cha MJ, Chung MJ, Lee KS, Kim TJ, Kim TS, Chong S, et al. Clinical features and radiological findings of adenovirus pneumonia associated with progression to acute respiratory distress syndrome: a single center study in 19 adult patients. Korean J Radiol. (2016) 17:9409. doi: 10.3348/kjr.2016.17.6.940

18. Ryu JS, Cho JH, Han HS, Jung MH, Yoon YH, Song ES, et al. Acute respiratory distress syndrome induced by adenovirus in an otherwise healthy woman. Yonsei Med J. (2003) 44:732-5. doi: 10.3349/ymj.2003.44.4.732

19. Narra R, Bono P, Zoccoli A, Orlandi A, Piconi S, Grasselli G, et al. Acute respiratory distress syndrome in adenovirus type 4 pneumonia: a case report. J Clin Virol. (2016) 81:78-81. doi: 10.1016/j.jcv.2016.06.005

20. Pfortmueller CA, Barbani MT, Schefold JC, Hage E, Heim A, Zimmerli S. Severe acute respiratory distress syndrome (ARDS) induced by human adenovirus B21: Report on 2 cases and literature review. J Crit Care. (2019) 51:9-104. doi: 10.1016/j.jcrc.2019.02.019

21. Centers for Disease Control and Prevention (CDC). Two fatal cases of adenovirus-related illness in previously healthy young adults-Illinois, 2000. MMWR Morb Mortal Wkly Rep. (2001) 50:553-5.

22. Centers for Disease Control and Prevention (CDC). Acute respiratory disease associated with adenovirus serotype 14-four states, 2006-2007. MMWR Morb Mortal Wkly Rep. (2007) 56:1181-4.

23. Chen X, Lv J, Qin L, Zou C, Tang L. Severe adenovirus pneumonia requiring extracorporeal membrane oxygenation support in immunocompetent children. Front Pediatr. (2020) 8:162. doi: 10.3389/fped.2020.00162

24. Engelmann I, Coiteux V, Heim A, Magro L, Dewilde A, Dulery R, et al. Severe adenovirus pneumonia followed by bacterial septicaemia: relevance of coinfections in allogeneic hematopoietic stem cell transplantation. Infect Disord Drug Targets. (2016) 16:69-76. doi: 10.2174/1871526516666160407114623

25. Hafidi M, Janin-Manificat H, Denis P, Charleux B, Rabilloud M, Boibieux A, et al. Acute retinal necrosis: virological features using quantitative polymerase chain reaction, therapeutic management, and clinical outcomes. Am J Ophthalmol. (2019) 208:376-86. doi: 10.1016/j.ajo.2019.08.007
26. Huh K, Kim I, Jung J, Lee JE, Jhun BW, Gu SH, et al. Prolonged shedding of type 55 human adenovirus in immunocompetent adults with adenoviral respiratory infections. Eur $J$ Clin Microbiol Infect Dis. (2019) 38:793-800. doi: 10.1007/s10096-019-0 3471-9

27. Albert E, Torres I, Bueno F, Huntley D, Molla E, Fernández-Fuentes MÁ, et al. Field evaluation of a rapid antigen test (Panbio ${ }^{\text {TM }}$ COVID-19 Ag Rapid Test Device) for COVID-19 diagnosis in primary healthcare centres. Clin Microbiol Infect. (2021) 27:472-7. doi: 10.1016/j.cmi.2020.11.004

28. Paiva JA, Laupland KB. Real -time PCR for early microbiological diagnosis: is it time? Intensive Care Med. (2017) 43:17146. doi: 10.1007/s00134-017-4793-1

29. Robert S, Lhommet C, Le Brun C, Garot D, Legras A, Mankikian J, et al. Diagnostic performance of multiplex PCR on pulmonary samples versus nasopharyngeal aspirates in community-acquired severe lower respiratory tract infections. J Clin Virol. (2018) 108:1-5. doi: 10.1016/j.jcv.2018.08.001

30. Boerger AC, Binnicker MJ. Comparison of the Panther Fusion respiratory panels to routine methods for detection of viruses in upper and lower respiratory tract specimens. Diagn Microbiol Infect Dis. (2020) 97:115014. doi: 10.1016/j.diagmicrobio.2020.115014

31. Shen Y, Cai G, Gong S, Dong L, Yan J, Cai W. Interaction between low tidal volume ventilation strategy and severity of acute respiratory distress syndrome: a retrospective cohort study. Crit Care. (2019) 23:254. doi: 10.1186/s13054-019-2530-6

32. Lynch JR, Kajon AE. Adenovirus: epidemiology, global spread of novel serotypes, and advances in treatment and prevention. Semin Respir Crit Care Med. (2016) 37:586-602. doi: 10.1055/s-0036-1584923

33. Ko JH, Lim JU, Choi JY, Oh HS, Yoo H, Jhun BW, et al. Early cidofovir administration might be associated with a lower probability of respiratory failure in treating human adenovirus pneumonia: a retrospective cohort study. Clin Microbiol Infect. (2020) 26:646-9. doi: 10.1016/j.cmi.2019.10.012

34. Maslo C, Girard PM, Urban T, Guessant S, Rozenbaum W. Ribavirin therapy for adenovirus pneumonia in an AIDS patient. Am J Respir Crit Care Med. (1997) 156:1263-4. doi: 10.1164/ajrccm.156.4.97-02028

35. Yoon BW, Song YG, Lee SH. Severe community-acquired adenovirus pneumonia treated with oral ribavirin: a case report. BMC Res Notes. (2017) 10:47. doi: 10.1186/s13104-016-2370-2

36. Ying B, Tollefson AE, Spencer JF, Balakrishnan L, Dewhurst S, Capella C, et al. Ganciclovir inhibits human adenovirus replication and pathogenicity in permissive immunosuppressed Syrian hamsters. Antimicrob Agents Chemother. (2014) 58:7171-81. doi: 10.1128/AAC.03860-14

Conflict of Interest: The authors declare that the research was conducted in the absence of any commercial or financial relationships that could be construed as a potential conflict of interest.

Publisher's Note: All claims expressed in this article are solely those of the authors and do not necessarily represent those of their affiliated organizations, or those of the publisher, the editors and the reviewers. Any product that may be evaluated in this article, or claim that may be made by its manufacturer, is not guaranteed or endorsed by the publisher.

Copyright (c) 2022 Wu, Zhang, Liu, Liu, Zhang, Zhang, Chen, He, Li, Xu and Liu. This is an open-access article distributed under the terms of the Creative Commons Attribution License (CC BY). The use, distribution or reproduction in other forums is permitted, provided the original author(s) and the copyright owner(s) are credited and that the original publication in this journal is cited, in accordance with accepted academic practice. No use, distribution or reproduction is permitted which does not comply with these terms. 